\title{
Ecdysone signaling is required for proper organization and fluid secretion of stellate cells in the Malpighian tubules of Drosophila melanogaster
}

\author{
NAVEEN-KUMAR GAUTAM and MADHU G. TAPADIA* \\ Cytogenetics Laboratory, Department of Zoology, Banaras Hindu University, Varanasi, India
}

\begin{abstract}
Drosophila development is a tightly regulated process involving metamorphosis of a relatively less mobile larva to a highly motile adult, triggered by secretion of 20-hydroxyecdysone. Under the influence of ecdysone, most of the larval tissues degenerate, while the imaginal cells differentiate and form adult specific structures. Although the larval Malpighian tubules do not seem to be affected by ecdysone during metamorphosis, we show that ecdysone signaling plays an important role in the early development and functioning of Malpighian tubules. Disruption of ecdysone receptor function, using targeted expression of dominant negative ecdysone receptor in stellate cells, results in disruption of organization of Malpighian tubules. The number of stellate cells is reduced in such Malpighian tubules. Further, they get clustered rather than distributed in their characteristic wild type pattern. We also demonstrate that expression of Drosophila integrin protein (DRIP), an aquaporin responsible for trans-cellular water transport, is also reduced in stellate cells when ecdysone signaling is disrupted. Our results show that of the three ecdysone receptor isoforms, only EcR-B2 rescues these phenotypes. A similar pattern of stellate cell clustering and reduced expression of DRIP is observed in ecd ${ }^{1}$, a temperature sensitive mutant, under non-permissive conditions. These results suggest that ecdysone signaling is required for proper patterning and functioning of stellate cells and that EcR-B2 may be the primary isoform required for ecdysone signaling in stellate cells.
\end{abstract}

KEY WORDS: Drosophila, ecdysone receptor, Malpighian tubule

\section{Introduction}

The Malpighian tubules (MTs) in Drosophilaare simple epithelial structures, which float in the hemocoel and which perform excretory and osmoregulatory functions analogous to vertebrate renal tubules. MTs comprise of two pairs of tubules which do not open to the outside, but are joined to alimentary canal at the junction of endodermal midgut and ectodermal hindgut. On the basis of the reporter gene expression in different enhancer trap lines, each pair of tubules has been divided into six segments, viz., the initial, transitional, main, lower tubule, upper ureter and lower ureter (Sozen et al., 1997). MTs are made up of two main cell types, the Type I or principal cells (PCs) and Type II or stellate cells (SCs) and four different minor cell types (Sozen et al., 1997). Recent studies have shown that the PCs and SCs of MTs originate from different cell lineages, with PCs originating from ectodermal epithelial buds in the hindgut and the SCs from the caudal mesoderm cells which lie above the hindgut in the region from where MTs bud (Denholm et al., 2003). The SCs undergo mesenchymal to epithelial transformation during intercalation

\footnotetext{
Abbreviations used in this paper: AF-1, activation function-1; AS, anterior spiracle; AP, anal plate; DAPI, 4, 6-diamidino-2 phenylindole dihydrochloride; DABCO, 1,4 diazobicyclo octane; DRIP, Drosophila integrin protein; EcR, ecdysone receptor; EcR-DN, dominant negative form of ecdysone receptor; Gal4, transcriptional activator protein in yeast; GFP, green fluorescent protein; MT, Malpighian tubules; PFA, paraformaldehyde; PBS, phosphate buffered saline; PCs, principal cells; RXR, retinoid X receptor; SCs, stellate cells; SG, salivary gland; USP, ultraspiracle; UAS, upstream activating sequence; VM, visceral masculature.
}

\footnotetext{
*Address correspondence to: Madhu Tapadia. Cytogenetics Laboratory, Department of Zoology, Banaras Hindu University, Varanasi, 221 005, India. Fax: +91-542-236-8457. e-mail: madhu@bhu.ac.in
}

Supplementary Material for this paper (one figure) is available at: http://dx.doi.org/10.1387/ijdb.092910ng

Accepted: 18 March 2009. Final author-corrected PDF published online: 23 February 2010. Edited by: Makoto Asashima.

ISSN: Online 1696-3547, Print 0214-6282 
and adopt epithelial characteristic once they are incorporated into the tubules and develop an apicobasal polarity. The primary function of MTs is secretion of isotonic fluid, which is accomplished by concerted action of physiologically distinct PCs and SCs. PCs are enriched with vacuolar- $\mathrm{H}^{+}-\mathrm{ATP}$ ase transporter which use $\mathrm{Na}^{+} / \mathrm{H}^{+}$and $\mathrm{K}^{+} / \mathrm{H}^{+}$exchanger to transport cations into the lumen (Davies et al., 1996; Dow et al., 1994, 1998; O'Donnell et al., 1996; Sozen et al., 1997) while SCs express channels which permit flow of $\mathrm{Cl}^{-}$ions (O'Donnell etal., 1998) and aquaporins which permit water flow into the lumen (Dow etal., 1995; Kaufmann et al., 2005). One of the Drosophila aquaporins, Drosophila Integral Protein (DRIP), a water specific aquaporin with very high transport rate is expressed in SCs of MTs (Kaufmann etal., 2005). Expression of DRIP is significantly high during embryonic and adult stages in SCs, which suggests that water moves transcellularly through them (Kaufmann et al., 2005).

MTs are unique in not undergoing extensive morphological changes during metamorphosis from larva to adult. Drosophila metamorphosis, which is coordinated by 20 -hydroxyecdysone (referred to as ecdysone) hormone and is characterized by diverse events resulting in degeneration of most of the larval tissues (Jiang et al., 1997) and proliferation and differentiation of imaginal cells into adult structures (Riddiford, 1993). Pulses of ecdysone also mark transition from one larval instar to the next.

Ecdysone binds to heterodimeric complex made up of Ecdysone Receptor (EcR) and Ultraspiracle (USP) (Koelle et al., 1992; Yao et al., 1992; Thomas et al., 1993) and up-regulates the primary response genes like Broad-complex, E74, E75, which code for transcriptional regulators for late response genes (Ashburner etal., 1974; Burtis et al., 1990; Thummel et al., 1990). The USPgene produces a single protein which is the Drosophila homologue of vertebrate RXR protein (Henrich et al., 1990; Oro et al., 1990). In contrast the ECR gene encodes three functional isoforms, EcR-A, EcR-B1 and EcR-B2 (Koelle et al., 1991; Talbot et al., 1993). These isoforms differ in their N-terminal, A/B or activation function-1 (AF-1) regions, but share a common conserved carboxy-terminal domain harboring the $C$ and $D$ domains referred to as the DNA and ligand binding regions respectively (Talbot et al., 1993). The unique AF-1 domains of EcR isoforms can activate genes in cell and target specific manner (Mouillet et al., 2001; Hu et al., 2003). Mutations that block all EcR isoforms are embryonic lethal (Bender et al., 1997), but mutations in any one of the isoforms affect specific developmental processes. EcR-A is predominantly expressed in tissues that develop into adult structures (Davis et al., 2005) while EcR-B1 predominantly expresses in larval structures which are destined to die (Bender et al., 1997; Schubiger et al., 1998). Due to the absence of EcRB2 specific mutations, the functions of this isoform are less understood. Ectopic expression of wild type EcRcan, to a certain extent, partially rescue $E c R$ mutants, depending upon the EcR isoform involved. It is still not clear how the MTs remain more or less refractory to ecdysone and are carried over to the adults when all other larval tissues are degraded or remolded under the influence of ecdysone.

In the present study we have examined the possible role of ecdysone signaling in the development and function of MTs. Since SCs are incorporated at a later stage during MT development and are functionally different from PCs, we were interested in knowing the role of ecdysone in incorporation and function of
SCs. We expressed a dominant negative mutant EcR (EcR-DN) (Cherbas et al., 2003) using the UAS-GAL4 system (Brand and Perrimon., 1993) in SCs of Malpighian tubules to block ecdysone signaling. Blocking ecdysone signaling using two GAL4 lines, c649 and $c 724$ results in developmental arrest at 1 st/2nd instar transition and $1^{\text {st }}$ instar, respectively. We show that the number of $\mathrm{SCs}$ is reduced in these mutants. We also show that the intercalation of SCs into the MTs during elongation is disrupted as these larvae show clusters of SCs, unlike the regular arrangement in wild type. Expression of Drosophila aquaporin, DRIP, is also reduced in these SCs. These results were confirmed using the $e c d^{\prime}$ temperature sensitive hypomorph allele, (Garen et al., 1977). In this case too, the SCs were irregularly distributed, with a reduced expression of DRIP. Co-expression of only EcR-B2 isoform with the EcR-DN in developing MTs, but not of EcR-B1 or EcR-A isoform, was able to rescue the lethal phenotype suggesting that EcR-B2 may be essential for SCs. A role of ecdysone in $\mathrm{SC}$ morphogenesis in MTs is further indicated by the fact that expression of EcR-DN with $c 724 \mathrm{Gal} 4$ driver which expresses in all the stellate cells results in a more drastic phenotype than with the $c 649 \mathrm{Gal} 4$ driver which expresses only in a subset of stellate cells.

\section{Results}

\section{Disruption of ecdysone receptor results in morphological changes in Malpighian tubules}

To examine the role of ecdysone in development of MTs, we used two dominant negative EcR proteins, $\mathrm{EcR}^{\mathrm{F} 645 \mathrm{~A}}$ and $\mathrm{EcR} \mathrm{R}^{\mathrm{W} 650 \mathrm{~A}}$, carrying mutations in the ligand binding domain of EcR and thus affecting all isoforms of ecdysone receptors (Cherbas et al., 2003). EcR ${ }^{\mathrm{F} 645 \mathrm{~A}}$ binds to ecdysone, while $E c R^{\mathrm{W} 650 \mathrm{~A}}$ does not, although both form heterodimers with USP and bind to the ecdysone response elements. Since they are not able to activate the target gene expression normally, the ecdysone signaling is interrupted when any one of them is expressed (Cherbas et al.,

TABLE 1

\section{EXPRESSION DOMAINS OF GAL4 DRIVERS USED IN THE PRESENT STUDY}

GAL4 Driver Domains of expression

$\begin{array}{ll}\text { c649 } & \begin{array}{l}\text { Bar- shaped stellate cell, salivary glands, proventriculus, mid gut, visceral } \\ \text { musculature cells, anterior spiracles, anal plate }\end{array} \\ \text { c724 } & \text { Stellate cells, bar shaped stellate cells, salivary glands, visceral musculature cells }\end{array}$

TABLE 2

\section{MEAN DIAMETER OF MALPIGHIAN TUBULES IS AFFECTED BY EXPRESSION OF ECR DN TRANSGENES}

\begin{tabular}{ll} 
Genotype & $\begin{array}{l}\text { Mean }(+\mathrm{S} . \mathrm{E} .) \text { diameter (in mm) of main segment } \\
(\mathrm{N}=30 \text { in each case) }\end{array}$ \\
\hline Wild type & $98.0 \pm 2.5$ \\
$c 649 \times E C R D N$ & $80.0 \pm 2.5^{*}$ \\
$c 724 \times E C R D N$ & $50.1 \pm 2.0^{*}$ \\
$c 649 \times E C R D N ; E C R$ B2 & $95.2 \pm 2.0$ \\
$c 724$ X ECR DN;ECR B2 & $93.0 \pm 2.8$ \\
\hline
\end{tabular}

* indicate significant differences in diameter of tubule $(P<0.05)$. 
2003). Since $E c R^{F 645 A}$ and $E c R^{W 650 A}$ exhibited identical results, we used $E c R^{F 645 A}$ in the present study and refer to it as EcR-DN in the following text. The EcR-DN was expressed in SCs of MTs with $c 649$ and 1724 Gal4 drivers. To identify the different tissues in which these Gal4 drivers are expressed, we used UAS GFP as reporter and observed the expression pattern (see supplementary Fig. S1). It was seen that besides the SCs, these drivers also express in a few other tissues (see Table 1). As shown in Table 1, only the SCs, visceral musculature and salivary glands are common tissues in which both these Gal4 drivers express. Expressing EcR-DN with c649and c724 Gal4 drivers resulted in slow development and larval lethality. The c649/t; EcR-DN/t (Fig. 1B) and $c 724 / E C R$-DN (Fig. 1C) larvae appeared smaller in size when compared to wild type larvae of similar age (Fig 1A). We looked at the MT as these drivers also express in SCs, we observed that the size of MTs from dying/ dead larvae of $c 649 /+; E C R-D N /+$ (Fig. $1 \mathrm{~B}$; blue arrow and Fig. $1 \mathrm{~b}$; black arrows) or $c 724 / E C R$ $D N$ (Fig.1C; blue arrow and Fig 1c; black arrows) was smaller when compared to wild type (Fig.1A; blue arrow and Fig 1a; black arrows). We measured the mean diameter of MTs (Table 2) from wild type across the main segment, which was $98.0 \pm 2.5 \mu \mathrm{m}$, while that in the $c 649 /+; E C R-D N /+$ and $c 724 / E C R-D N$ larvae was $80.0 \pm 2.5 \mu \mathrm{m}$ and $50.1 \pm 2 \mu \mathrm{m}$, respectively. Larvae from $c 724 / E C R-D N$ showed greater reduction in size than $c 649 /+; E c R-D N /+$. Larvae from these crosses also exhibited other gross abnormalities, like irregular tracheae (Fig. $1 \mathrm{~B}$ and $1 \mathrm{C}$; red arrow), low adipose tissue, and sluggish movements. Cuticular preparations of dying/dead larvae in these crosses showed defects in larval molting. The cuticular preparation of $c 649 /+; E c R-D N /+$ larvae (Fig. 2B) revealed the presence of both $1^{\text {st }}$ (Fig. 2B; black arrows) and $2^{\text {nd }}$ (Fig. 2B; red arrow) instar larval mouth hooks, suggesting that these larvae retain the cuticle from previous instar in addition to their newly formed cuticle while the control wild type larvae had already reached $3^{\text {rd }}$ instar stage as revealed by the mouth hooks (Fig 2A; black arrow). Cuticular preparations from $c 724 / E C R$-DNdying larvae (Fig. 2C; black arrows), on the other hand, showed mouth hooks characteristic of first instar stage suggesting their death during $1^{\text {st }}$ instar itself.

\section{Disruption of ecdysone receptor leads to reduction in the number of stellate cells}

A remarkable feature of $\mathrm{MT}$ is that the number of PCs and the SCs remain fairly constant throughout development (Wessing and Eichelberg, 1978; Sozen et al., 1997). Since following expression of EcR-DN, the MTs were smaller in size, we counted the number of different cell types in MTs from wild
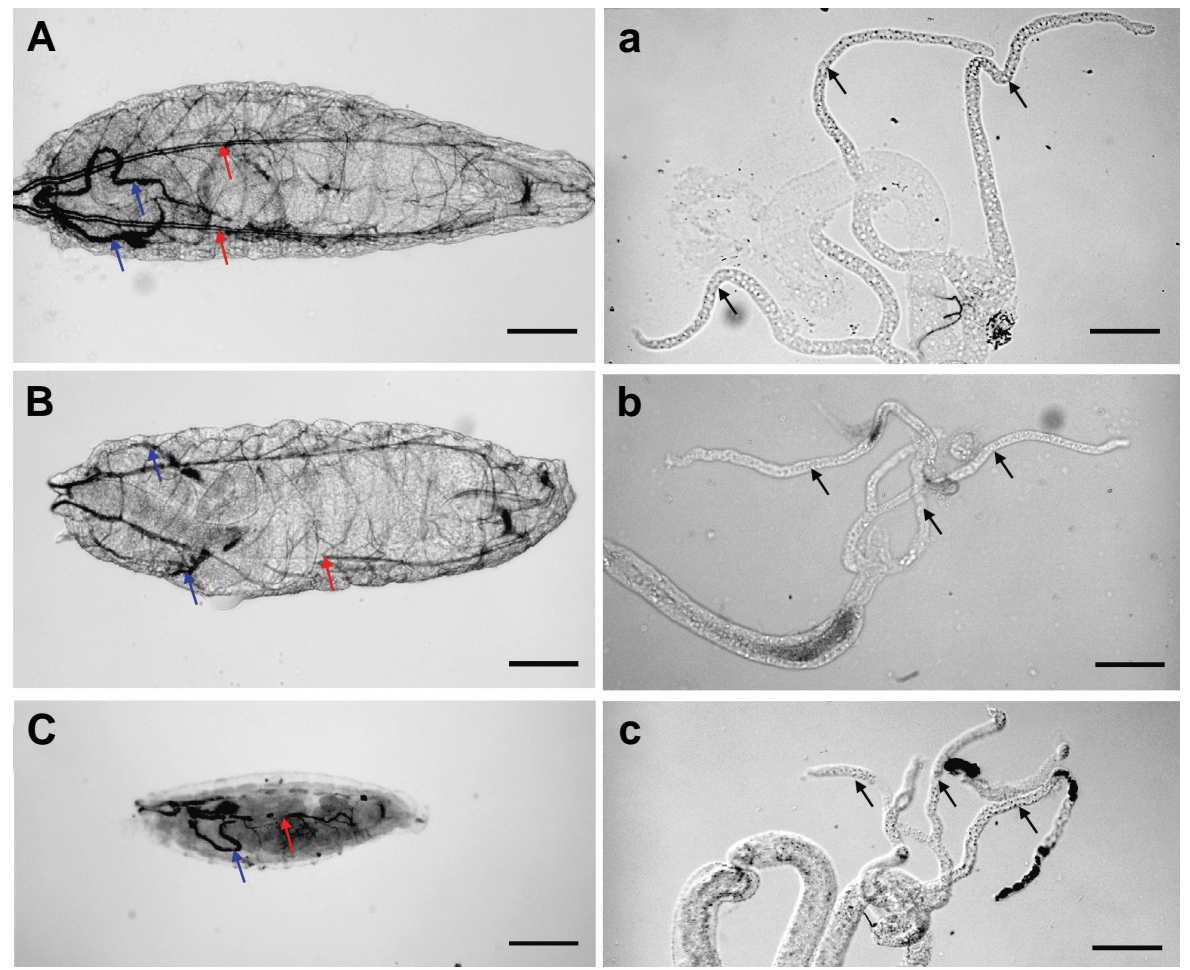

Fig. 1. Developmental defects of larvae and morphology of Malpighian tubules (MT). Delayed development of larva from 649/+; ECR-DN/+ (B), c724/ECR-DN (C) when compared to wild type (A). MTs and trachea are indicated by blue arrow and red arrows respectively. Malpighian tubules of wild type (a) are developed completely when compared to c649/+; EcR$D N /+$ (b) and c724/EcR-DN (c). Bar represents $100 \mu \mathrm{m}$ in each case.

type, $c 649 /+; E c R-D N /+$ and $c 724 / E c R$-DNlarvae. For an unambiguous identification of the SCs, the MTs were immuno-stained with teashirt antibody, which is a specific marker for SCs (Jung et al., 2005; Singh et al., 2007). Data in Table 3 show that the numbers of PCs in the anterior and posterior tubules of $c 649 /+$; $E C R-D N /+$ and $c 724 / E c R-D N g$ enotypes were similar to that in the wild type. However, while the mean numbers of SCs in the wild type anterior and posterior tubules were $31.8 \pm 0.2$ and $21.4 \pm 0.1$, respectively, those in anterior and posterior tubules of $c 649 /+$; $E C R-D N /+$ larvae were $24.9 \pm 0.4$ and $17.5 \pm 0.2$, respectively and in $c 724 / E c R$-DNMTs, these were $18.5 \pm 0.6$ and $13.7 \pm 0.4$, respec-
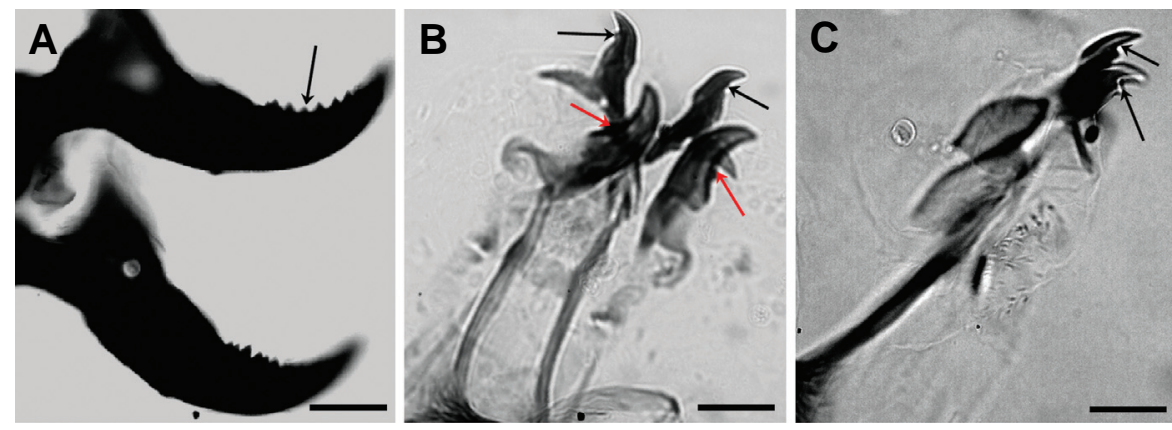

Fig. 2. Larval cuticular preparations. Showing $3 r d$ instar mouth-hooks of wild type (A), $1^{\text {st }}$ and $2^{\text {nd }}$ instar mouth hooks of c649/+; EcR-DN/+ (B) and $1^{\text {st }}$ instar mouth hooks of c724/EcR-DN (C). Black arrows represent the $1^{\text {st }}$ instar and red arrows show the $2^{\text {nd }}$ instar larval mouthhooks. Bar represents $20 \mu \mathrm{m}$. 

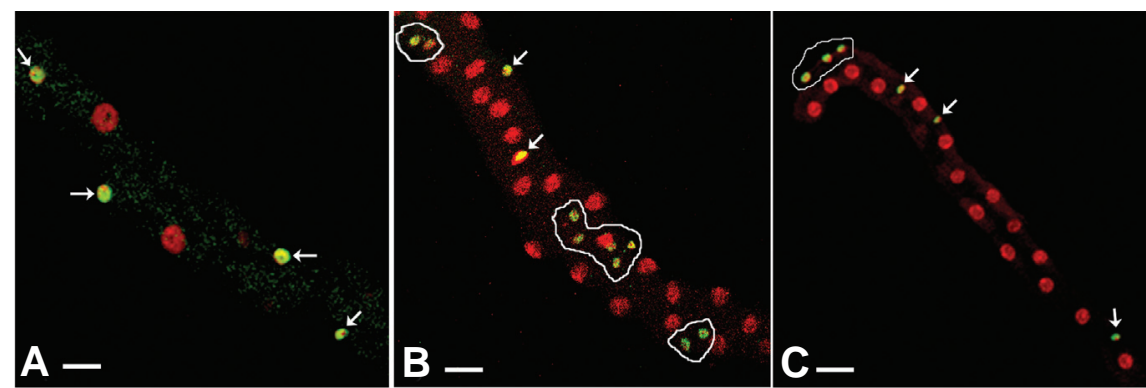

Fig. 3. Aberrant intercalation of stellate cells. $E X-$ pression of EcR-DN in SCs using c649 (B) and c724 (C) drivers leads to clustering of these cells when compared to regular arrangement of wild type (A) Coexpression of ECR-B2 rescues the clustering phenotypes induced by EcR-DN when driven by c649 (D) and c724 (E) SCs are identified by anti- teashirt antibody (green fluorescence) and chromatin is stained with DAPI (Pseudocolour red). Arrows indicate single stellate cells while encircled area shows clustering of stellate cells. Bar represents $20 \mu \mathrm{m}$.

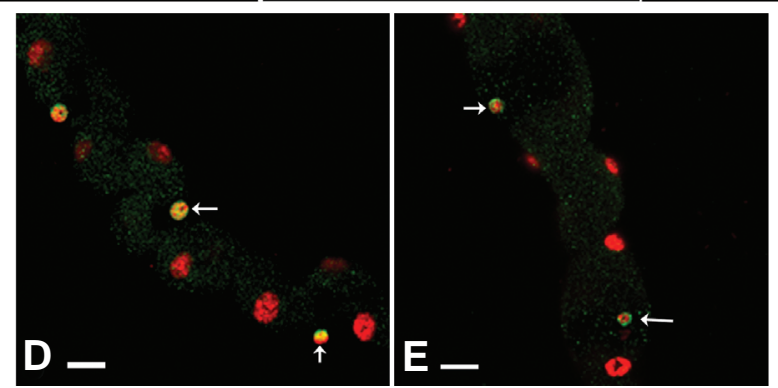

tively. We subjected this data to one way anova followed by post hoc test and found that the number of SCs in $c 649 /+; E c R-D N /+$ and $c 724 / E C R-D N$ was significantly reduced when compared to wild type $(\mathrm{p}<0.05)$. It was also seen that the $c 724 \mathrm{Gal} 4$ driver caused greater reduction in numbers of SCs than the 649 Gal4 driver (Table 3).

\section{Disruption of ecdysone signaling leads to aberrant pattern of integration of stellate cells}

Drosophila MTs are derived from the ectodermal epithelial buds and the mesenchymal mesodermal tissues (Denholm et al., 2003). During embryogenesis, the principal cells are formed by stage 13 (Skaer, 1989). The SCs are found near the vicinity of MTs at stage 11/12 and at stage 13 the SCs have just started intercalation, but only by stage 15 the SCs are fully incorporated in the MTs (Denholm et al., 2003). SCs are distributed evenly throughout the anterior and posterior tubules at regular intervals (Sozen et al., 1997). Ecdysone plays an important role in embryonic morphogenesis. Maternally deposited ecdysteroids are the source of active ecdysone (Hoffman et al., 1985), however till stage 11 no ecdysone activity is observed. Ecdysone activity is first observed at stage 12 and peaks at stage 13 and continues to be high after that (Kozlova and Thummel, 2003). Since ecdysone activity is first observed just prior to SCs intercalation, it is very likely that this process is governed by ecdysone. We investigated the role of ecdysone in arrangement of the SCs. In wild type larvae (Fig. 3A), the SCs were distributed in the characteristic pattern as reported earlier (Sozen et al., 1997). However, in larvae expressing EcR-DN under the c649(Fig. 3B) and the c724(Fig. 3C) Gal4 driver, the regular arrangement of SCs in MTs was lost, instead they were found to be present in clusters suggesting that the intercalation of SCs into the MTs during embryogenesis was disrupted. We counted the number of SC clusters in wild type and compared them with EcR-DN mutant expressing MTs (Table 4). It was found that in 25 wild type MTs examined, there were only five instances when SCs were present in clusters, whereas in similar numbers of $c 649 / 4 ; E c R-D N /+$ and $c 724 / E c R-D N M T s$, majority of the tubules showed clusters of SC (Table 4). Further, the number of SCs in each cluster in wild type was never more than two, but the clusters in EcR-DN expressing MTs showed

TABLE 3

THE NUMBER OF STELLATE CELLS IS REDUCED IN ECR-DN EXPRESSING MALPIGHIAN TUBULES

\begin{tabular}{|c|c|c|c|c|}
\hline \multirow[b]{2}{*}{ Genotype (N=30 in each case) } & \multicolumn{2}{|c|}{ Principle cells } & \multicolumn{2}{|c|}{ Stellate cells } \\
\hline & Anterior tubule & Posterior tubule & Anterior tubule & Posterior tubule \\
\hline Wild type & $142.9 \pm 0.3$ & $110.0 \pm 0.3$ & $31.8 \pm 0.2$ & $21.4 \pm 0.1$ \\
\hline c649X EcR DN & $140.0 \pm 0.6$ & $104.1 \pm 0.8$ & $24.9 \pm 0.4^{*}$ & $17.5 \pm 0.2^{*}$ \\
\hline c649X EcR DN;EcR B2 & $142.4 \pm 0.4$ & $106.3 \pm 0.5$ & $28.5 \pm 0.4$ & $19.8 \pm 0.3$ \\
\hline$c 724 X E C R D N$ & $141.9 \pm 0.4$ & $109.3 \pm 0.5$ & $18.5 \pm 0.6^{*}$ & $13.7 \pm 0.4^{\star}$ \\
\hline$c 724 X$ EcR DN; EcR B2 & $141.1 \pm 0.5$ & $102.4 \pm 0.9$ & $27.5 \pm 0.6$ & $20.2 \pm 0.2$ \\
\hline
\end{tabular}

* shows significantly reduced stellate cells number $(\mathrm{P}<0.05)$.

TABLE 4

THE NUMBER OF STELLATE CELL CLUSTERS AND THEIR RANGE IN ANTERIOR AND POSTERIOR TUBULES

\begin{tabular}{|c|c|c|c|c|}
\hline \multirow[b]{2}{*}{ Genotype } & \multicolumn{2}{|c|}{ Anterior tubules $(\mathrm{N}=25)$} & \multicolumn{2}{|c|}{ Posterior tubule $(\mathrm{N}=25)$} \\
\hline & Total No. of Clusters & Range of cells in a cluster & Total No. of Clusters & Range of cells in a cluster \\
\hline WT & 6 & 2 & 4 & 2 \\
\hline$c 649 \times E C R D N$ & $128^{*}$ & $2-6^{*}$ & $25^{*}$ & $2-3^{*}$ \\
\hline$c 724 \times E c R D N$ & $95^{*}$ & $2-5^{*}$ & $52^{*}$ & $2-4^{*}$ \\
\hline$c 649 \times$ EcR DN; EcR B2 & 7 & 2 & 4 & 2 \\
\hline$c 724$ X EcR DN; EcR B2 & 10 & 2 & 8 & 2 \\
\hline
\end{tabular}

$\mathrm{N}$ - total number of MTs counted. * shows the significant increase in cluster number and Size $(\mathrm{P}<0.05)$. 
between two to seven SCs per cluster.

\section{The B2 ecdysone receptor isoform is required for maintain-} ing normal stellate cell number and integration

The three EcR isoforms are known to be involved in distinct functions depending upon their spatial and temporal expression (Kim etal., 1999; Snug and Robinow., 2000; Truman et al., 1994). It is known that expression of wild type EcR can to a certain extent, rescue phenotypes resulting from EcR-DN expression (Schubiger, 2003; Cherbas et al., 2003). Therefore, we individually co-expressed each of the wild type EcR isoforms (EcR-B1, EcR-B2 or EcR-A) along with EcR-DN to examine possible rescue. To find out if over-expression of EcR-B1, EcR-B2 or EcR-A with any of the two Gal4 drivers by itself had any detectable phenotype, we expressed each of these isoforms in the wild type background. We did not observe any effect on the viability and phenotype following over-expression of any of these EcR isoforms in the target tissues (data not shown). Then we co-expressed each of these isoforms individually with EcR-DN using the two Gal4 drivers as above. It was found that expression of EcR-B2 isoform only was capable of rescuing the lethality caused by EcR-DN. The morphology and pattern of intercalation of SCs in MTs co-expressing EcR-DN and EcR-B2 under the $c 649$ (Fig. 3D) or the c724(Fig. 3E) drivers was similar to that in wild type. The numbers of SCs in EcR-B2 co-expressing in MTs were restored to almost to the level in wild type (Table 3). Likewise the mean diameter of these MTs was also found to be not significantly different from that in wild type (Table 2). Coexpression of EcR-B1 and EcR-A along with EcR-DN with any of the two Gal4 drivers, however, was not able to suppress the altered MT phenotype (data not shown).

\section{Expression of dominant negative form of the ecdysone receptor reduces DRIP expression in stellate cells}

Expression of DRIP, which facilitates water transport (Dow etal., 1995, Kaufmann et al., 2005), has been reported only in the SCs during embryogenesis and in adults (Kaufmann et al., 2005). To investigate if DRIP is expressed in larvae and if ecdysone signaling is required for DRIP expression, we immunostained wild type larval MTs using anti-DRIP antibody. It was seen that as in embryos and adults (Kaufmann et al., 2005), DRIP is also expressed in the larval MTs and the expression is limited to only SCs (Fig. 4A). Expression of DRIP was greatly reduced in SCs following expression of EcR-DN under the $c 649$ (Fig. 4B) or c724Gal4 driver (Fig. 4C). For exact comparison we measured DRIP intensity using Profile Display Function of Zeiss LSM

a
Meta 510 software which displays the intensity of flurochrome. The fluorescence intensity of DRIP and DAPI is depicted on the $Y$ axis and the marked line in Fig. 4 (A,B,C,D) starting from tail to arrow head and is plotted on the $X$ axis. We found that the intensity of DRIP (Green colour graph) in c649/+; EcR-DN/+ (Fig. 4b) and $c 724 / E C R$-DN(Fig. 4c) was much less than wild type (Fig. 4a). Coexpression of EcR-B2 in MTs expressing EcR-DN under c649 (Fig. 4E,e) and $c 724$ (Fig. 4F,f) Gal4 driver restored the DRIP expression to near wild type levels. As noted with MT morphology, co-expression of $E C R$-B1 or $E C R$-Adid not elevate the expression of DRIP in SCs (data not shown).

\section{Malpighian tubules of larvae homozygous for temperature- sensitive ecd ${ }^{1}$ mutant allele exhibit reduced stellate cell numbers and defects in stellate cell intercalation at non- permissive temperature}

$e c d^{1}$ (Garen et al., 1977) is a temperature sensitive allele for
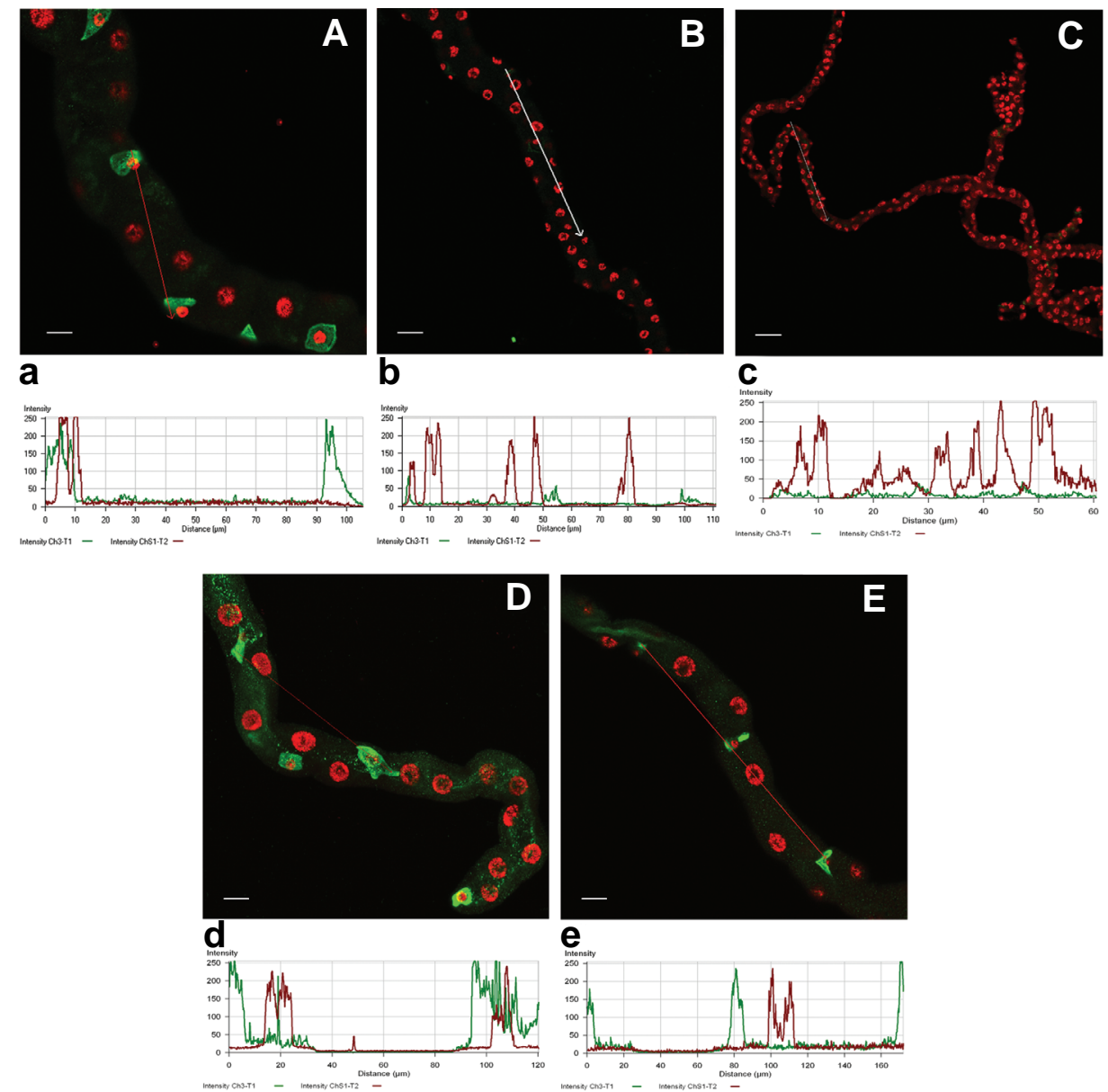

Fig. 4. Drosophila integrin protein (DRIP) levels are reduced in stellate cells when EcR-DN is expressed. Compared to wild type (A), DRIP expression is less in c649/+; EcR-DN/+ (B) and c724/ $E_{C} R-D N(\mathbf{C})$. Coexpression of ECR-B2 restores DRIP expression to nearly wild type levels when driven by c649 (D) and c724 (E). DRIP is labeled with FITC (green fluorescence) and chromatin with DAPI (pseudocolour red). The graph below each slide $(\mathbf{a}, \mathbf{b}, \mathbf{c}, \mathbf{d}, \mathbf{e})$ represents the Profile display function of LSM 510 Meta which measures the intensity of fluorescence along the line drawn. In Graph green colour shows intensity of DRIP while red shows the DAPI. Bar represents $20 \mu \mathrm{m}$ in each case. Arrows passing through different cells show DRIP expression only in stellate cells and intensity in these cells is measured by the Profile display function. 
ecdysone production and is unable to synthesize ecdyosne when shifted to $29^{\circ} \mathrm{C}$. It's a hypomorph allele showing lethality at nonpermissive conditions. In order to confirm if the observed phenotypes following disruption of EcR is because of ecdysone signaling, we grew ecd homozygous embryos at non-permissive temperature, and examined the arrangement of SCs in MTs from $1^{\text {st }}$ instar larvae. The SCs in these MTs were indeed clustered (Fig. $5 A$ ) similar to that seen when EcR-DN is expressed in MTs (Fig. 3). The expression of DRIP in these SCs was also found to be reduced (Fig. 5D) than in wild type (Fig. 4A). The fluorescence intensity profile of DRIP also showed reduced intensity levels (Fig. 5d,f).

\section{Discussion}

In Drosophila, developmental events like molting at different larval instars and metamorphosis during pupal period is triggered by the ecdysteroids. Malpighian tubules is an interesting tissue to study developmental biology, because firstly it does not undergo ecdysone induced degeneration (Jiang et al., 1997) and secondly it is made of cells from two different lineages (Denholm et al., 2003) which have physiologically distinct functions (Davies et al., 1996; Dow et al., 1994, 1998; O'Donnell et al., 1996; Sozen et al., 1997; O'Donnell etal., 1998). Though ecdysone signaling in larval
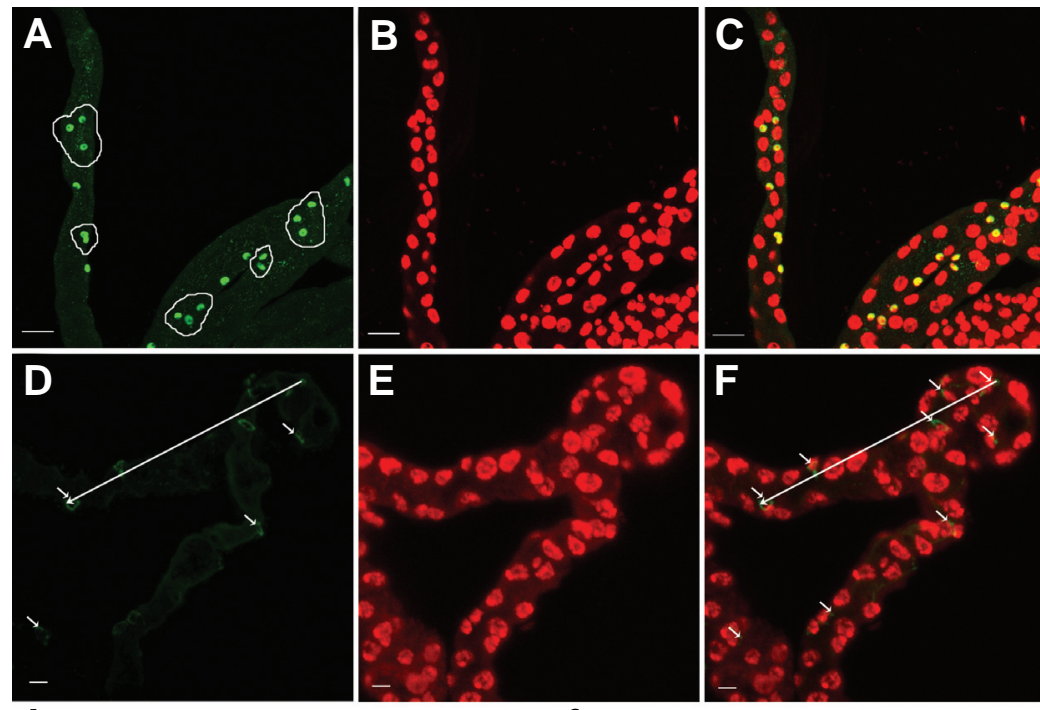

d

f
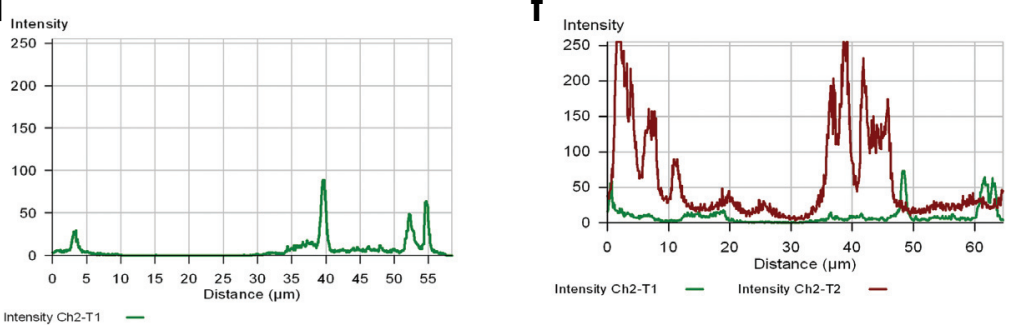

Fig. 5. Ecdysone levels affect stellate cell organization and Drosophila integrin protein (DRIP) levels. Immunostaining with anti-teashirt antibody in ecd ${ }^{1}$ mutant shows clusters of SCs (A), DAPI (B) and merged (C). ecd mutants also show reduced expression of DRIP(D), DAPI (E) and merged (F). The intensity of DRIP(d,f) is measured by Profile display function of LSM Meta 510 software. DAPI is pseudocolour red. Bar represents $50 \mu \mathrm{m}(A, B, C)$ and $5 \mu \mathrm{m}$ in $(D, E, F)$.
MTs does not lead to cell death, we find that disruption of ecdysone signaling using EcR-DNin SCs during early development results in improper morphogenesis of MTs which affects their functioning as reflected in the reduced DRIP expression. These results clearly suggest that ecdysone has an important role in development and physiology of MTs. Earlier studies on the expression of ECR-DN with Eip, GMR, Ser or $d p p$ Gal4 drivers revealed local as well as a global effects resulting in complete blockage of development (Cherbas et al., 2003) suggesting that there is a molting checkpoint. It is likely that localized malfunctioning of EcR in important tissues is sensed by the surveillance system and in cases of irreparable defects, blocks development at the next ecdysone dependent event. Our data suggest that EcR dependent functioning of SCs in MTs could also be one of the critical functions whose malfunctioning can ultimately lead to larval death.

Our present studies show a new role of ecdysone in proper intercalation of SCs. The normal incorporation of SCs depends on the Hibris protein, which is an ortholog of mammalian protein NEPHRIN. Hibris helps to stabilize the intercalation of SCs in MTs (Denholm et al., 2003, Artero et al., 2006). Hibris acts in cell-cell recognition and attraction during embryogenesis (reviewed by Chen and Olson, 2004). We show that ecdysone signaling affects SC arrangement, leading to the possibility that ecdysone may be regulating hibris expression either directly or indirectly. Since disruption of ecdyosne signaling in SCs does not affect PC integration our present results further suggest that the arrangement of SCs and PCs are independent of each other. The arrangement of PCs is largely controlled by the product of rols, since in MTs of rols mutants while the PCs were in clusters; the SCs were normally integrated between the PCs (Putz et al., 2005). Our results show that the intercalation requires ecdysone at some stage and that the EcR-B2 isoform may be the major isoform through which ecdysone signaling is transduced. It is notable in this context that migration of border cells in egg chambers requires $\mathrm{C} /$ EPB transcription factor encoded by slow border cells (s/bo) locus and in slbo mutants, border cells migrate either very slowly or not at all (Rorth et al., 1998). Expression of EcR-DN driven by s/bo-GAL4 in ovaries, results in failure of border cells migration which is subsequently rescued by the expression of EcR-B2 isoform (Cherbas et al., 2003). In view of our present results, it is tempting to speculate that the developmental migration of different cells is triggered by ecdysone and EcR-B2 may be the key isoform that tranduces the signal.

SCs play an important role in fluid secretion and inhibition of chloride channels affects functioning of SCs and ultimately affects fluid secretion (Sozen et al., 1997). In hibris mutants, where the number of SCs is reduced, the fluid transport is also affected, resulting in lethality (Denholm et al., 2003). In agreement with some early reports that ecdysone plays a role in fluid secretion by altering membrane permeability (Gee et al., 1977; Ryerse, 1978), we provide direct evidence that ecdysone, directly or indirectly controls the fluid secretion of SCs via its influence on the expression of 
DRIP.

Stellate cells have an important role and the appropriate number of SC is essential for proper development. This is evidenced by the fact that the phenotypes derived from $c 724$ are more drastic than similar phenotypes observed by $c 649$. This could be because $c 724$ affects the entire stellate cells, where as the $c 649$ affects only a small proportion of bar shaped stellate cells.

Our present results thus show that proper ecdysone signaling via the EcR-B2 in MTs during embryonic development is required for correct integration of SCs in developing MTs and for their physiological functions involving DRIP expression.

\section{Materials and Methods}

\section{Drosophila stocks}

Following fly stocks used in the present study were obtained from the Bloomington stock centre unless mentioned otherwise. Wild type Oregon $\mathrm{R}^{+}$, stellate cells specific GAL4 drivers $c 724$ and $c 649$ (Kind gift by Dr. J. A. T. Dow, Institute for Biomedical Sciences, University of Glasgow, UK), UAS responders, $P\left\{U A S\right.$-ECR.B1-DeltaC655. F645A\} (ECR $\left.{ }^{F 645 A}\right) P\{U A S$ ECR.B1-DeltaC655.W650A\} (ECRW650A) P\{UAS-ECR.B1\} (ECR-B1), P\{UAS-ECR.B2\} (ECR-B2), P\{UAS-ECR.A (ECR-A). Temperature sensitive hypomorph allele for ecdysone $\left(e c d^{1} s t^{1} \mathrm{red}^{1} e^{4} c a^{1}\right)\left(e c d^{\prime}\right)$ (Garen et al., 1977) was obtained from Dr. C. Thummel (Department of Human Genetics, University of Utah School of Medicine, UT). Appropriate crosses were set up to generate P\{UAS-ECR.B1-DeltaC655.F645A\}; P\{UASECR.B1\}, P\{UAS-ECR.B1-DeltaC655.F645A\};P\{UAS-ECR.B2\}, P\{UASECR.B1-DeltaC655.F645A; $\{\{\cup A S-E C R$.A\}. The references to these stocks in the paper are shown in bracket and bold. All flies and larvae were reared at $25 \pm 1^{\circ} \mathrm{C}$ on standard food containing maize powder, agar, dried yeast and sugar at 12 hours dark: 12 hours light cycle. For checking lethality, egg laying was set on agar plates and the number of eggs laid was counted. After hatching they were counted and checked for the stage of lethality. In the rescue experiments, similar procedure was done and finally the number of adults emerged was used to calculate the percentage of rescue. The $e c d^{\prime}$ flies were allowed to lay eggs at $25 \pm 1^{\circ} \mathrm{C}$ and then shifted at $29^{\circ} \mathrm{C}$ for further development.

\section{Morphological analysis of larvae and Malpighian tubules}

Larvae of different genotypes were collected from food plates and washed with distilled water. They were anaesthetized with ether and mounted on bridged slides in $50 \%$ glycerol and observed under bright field. The MTs were dissected in 1XPBS (18.6mM NaH $\mathrm{PO}_{4}, 84.1 \mathrm{mM}$ $\mathrm{Na}_{2} \mathrm{HPO}_{4}, 17.5 \mathrm{mM} \mathrm{NaCl}$ ) from larvae of desired genotype and mounted in $50 \%$ glycerol and observed under DIC optics. Images were collected using Nikon Digital camera DXM 1200 fitted on the Nikon E800 microscope. For measuring the diameter Malpighian tubules, they were dissected and spread on a slide. The diameter at the main segment was measured with an ocular micrometer under 10X magnification in units of $16.7 \mu \mathrm{m}$. For examining the cuticle, mouth-hooks and spiracles, larvae of appropriate genotypes were washed in 1X PBS, fixed in 1:4 glycerols: acetic acid and incubated over-night at $60^{\circ} \mathrm{C}$. They were mounted in Hoyer's mounting medium covered with coverslips under a $20 \mathrm{gm}$ weight and incubated again at $60^{\circ} \mathrm{C}$ for 2 days. Images were captured as above.

\section{Immunocytochemistry}

Malpighian tubules from larvae of appropriate genotypes were dissected in $1 \mathrm{X}$ PBS, fixed in $4 \%$ formaldehyde for $20 \mathrm{~min}$ at $\mathrm{RT}$, rinsed in PBST (PBS, $0.1 \%$ Triton $\mathrm{X}-100$ ) and processed for immunostaining essentially as described earlier (Patel NH,1994). The MTs were mounted in antifadant, DABCO (Sigma). The primary antibodies used were antiteashirt used at 1:3000 and anti-DRIP used at 1:1000. Goat-anti rabbit AF488 (Molecular probes, USA) was used as secondary antibody. Chro- matin was stained with DAPI $(1 \mu \mathrm{g} / \mathrm{ml}$ in 1 XPBS). All preparations were analyzed on a Ziess LSM 510 Meta Confocal microscope and images were processed with Adobe Photoshop.

\section{Acknowledgements}

We thank Bloomington Stock Center for fly stocks. We are thankful to Dr. J. A. T. Dow and Dr. C. Thummel for fly stocks and for their useful suggestions. We also thank Dr. S. Cohen, EMBL Heidelberg, Germany and Dr. N. Kauffmann, University of Pittsburgh, Pennsylvania, for kindly providing anti-teashirt and anti-DRIP antibodies respectively. We are very thankful to Prof S. C. Lakhotia for his critical comments and valuable inputs in the paper. We are thankful to Department of Science and Technology, Government of India, New Delhifor financialsupport to MGT and also for the Multiphoton Confocal Microscope National Facility. Financial support to NKG from CAS, Department of Zoology, BHU is greatly acknowledged.

\section{References}

ARTERO, R.D., MONFERRER, L., GARCIA-LOPEZ, A. and BAYLIES, M.K. (2006). Serpent and a hibris reporter are co-expressed in migrating cells during Drosophila hematopoiesis and Malpighian tubule formation. Herediats 143: 141-157.

ASHBURNER, M. (1974). Sequential gene activation by ecdysone in polytene chromosomes of Drosophila melanogaster. II. The effects of inhibitors of protein synthesis. Dev. Biol. 39: 141 -157.

BENDER, M., IMAM, F.B., TALBOT, W.S., GANETZKY, B. and HOGNESS, D.S (1997). Drosophila ecdysone receptor mutations reveal functional differences among receptor isoforms. Cel/91: 777- 788.

BRAND, A.H. and PERRIMON, N. (1993). Targeted gene expression as a means of altering cell fates and generating dominant phenotypes. Development 118 : 401-415.

BURTIS, K.C., THUMMEL, C.S., JONES, C.W., KARIM, F.D. and HOGNESS, D.S (1990). The Drosophila 74EF early puff contains E74, a complex ecdysoneinducible gene that encodes two ets-related proteins. Ce//61: 85-99.

CHEN, E.H. and OLSON, E.N. (2004). Towoards a molecular pathway of myoblast fusion in Drosophila. Trends in Cell Biol14: 452-460.

CHERBAS, L., HU, X., ZHIMULEV, I., BELYAEVA, E. and CHERBAS, P. (2003). $\mathrm{EcR}$ isoforms in Drosophila: testing tissue-specific requirements by targeted blockade and rescue. Development 130: 271-284

DAVIS, M.B., CARNEY, G.E., ROBERTSON, A.E. and BENDER, M. (2005) Phenotypic analysis of $E c R A$ mutants suggests that $E c R$ isoforms have unique functions during Drosophila development. Dev. Biol. 282:385-396.

DAVIES, S.A., GOODWIN, S.F., KELLY D.C., Wang, Z., SOZEN, M.A., KAISER, K. and DOW, J.A.T. (1996). Analysis and Inactivation of vha55, the gene encoding the Vacuolar ATPase B- subunit in Drosophila melanogaster, reveals a larval lethal phenotype. J. Biol. Chem. 271: 30677-30684.

DENHOLM, B., SUDARSAN, V., PASALODOS-SANCHEZ, S., ARTERO, R., LAWRENCE, P., MADDRELL, S., BAYLIES, M. and SKAER, H. (2003). Dual origin of the renal tubules in Drosophila: mesodermal cells integrate and polarize to establish secretory function. Curr. Biol. 13: 1052-1057.

DOW, J.A.T., KELLY, D.C., DAVIES, S.A., MADDRELL, S.H.P. and BROWN, D. (1995). A novel member of the major intrinsic protein family in Drosophila: are aquaporins involved in insect Malpighian (renal) tubule secretion? J. Physiol. 489:110-111.

DOW, J.A.T., MADDRELL, S.H.P., GOERTZ, A., SKAER, N.J.V., BROGON, S. and KAISER, K. (1994). The Malpighian tubules of Drosophila melanogaster. a novel phenotype for studies of fluid secretion and its control. J. Exp. Biol. 197: 421-428.

DOW J.A.T., DAVIES, S.A. and SOZEN, M.A. (1998). Fluid secretion by the Drosophila Malpighian tubule. Am. Zool. 38: 450-460.

GAREN, A., KAUVAR, L. and LEPESANT, J-A. (1977). Role of ecdysone in Drosophila development Proc. Natl. Acd. Sci. USA 74: 5099-5103.

GEE, J.D. and WHITEHEAD, D.L. (1977). Steroids stimulate secretion by insect Malpighian tubules. Nature 269: 238-239. 
HELEN SKAER. (1989). Cell division in Malpighian tubule development in $D$. melanogaster is regulated by a single tip cell. Nature 342: 566-569.

HENRICH, V.C., SLITER, T.J., LUBAHN, D.B., MACINTYRE, A. and GILBERT, L.I. (1990). A Steroid/thyroid hormone receptor superfamily member in Drosophila melanogaster that shares extensive sequence similarity with a mammalian homologue. Nucleic Acids Res. 18: 4143-4148.

HOFMANN, J.A., LAGUEUX, M. (1985). Endocrine aspect of embryonic development in insect. In Comprehensive insect physiology, biochemistry and pharmacology (Ed. G.A. Kerkut and L.I. Gilbert) Pergamon Press, Oxford 1: 435-460.

HU, X., CHERBAS, L. and CHERBAS, P. (2003). Transcription activation by the ecdysone receptor (EcR/USP): Identification of activation function. Mol. Endocrinol. 17: 716-731.

JIANG, C., BAEHRECKE, E.H. and THUMMEL, C.S. (1997). Steroid regulated programmed cell death during Drosophila metamorphosis. Development 124: 4673-4683.

JUNG, C. A., DENHOLM, B., SKAER, H. and AFFELTER, M. (2005). Renal tubule development in Drosophila: A closer look at cellular level. J. Am. Soc. Nephrol. 16: 322-328.

KIM S.J., PARK, J.G. and LEE, C.C. (1999). Transcript titers of ecdysteroid receptor components vary between tissues and stages during Drosophila development. Mol. Cells 9: 61-66.

KAUFMANN, N., MATHAI, J.C., HILL, W.G., DOW, J.A.T., ZEIDEL, M.L. and BRODSKY, J.L. (2005). Developmental expression and biophysical characterization of a Drosophila melanogasteraquaporin. Am. J. Physiol. Cell. Physiol. 289: 397- 407.

KOELLE, M.R., TALBOT, W.S., SEGRAVES, W.A., BENDER, M.T., CHERBAS, P. and HOGNESS, D.S. (1991). The Drosophila EcR gene encodes an ecdysone receptor, a new member of the steroid receptor super family. Cel/67:59-77.

KOELLE M.R, SEGRAVES W.A. and HOGNESS D.S. (1992). DHR3: a Drosophila steroid receptor homolog. Proc. Natl. Acad. Sci. USA 89: 6167-6171.

MOUILLET J.-F., HENRICH V.C., LEZZI M. and VOGTLI M. (2001) Differential control of gene activity by isoforms A, B1, and B2 of the Drosophila ecdysone receptor. Eur. J. Biochem 268: 1811-1819.

O'DONNELL M.J., RHEAULT M.R., DAVIES S.A., ROSAY P., HARVEY B.J., MADDRELL S.H.P., KAISER K. and DOW J.A.T. (1998). Hormonally controlled chloride movement across Drosophila tubules is via ion channels in stellate cells. Am. J. Physiol. 73: 1039-1049.

O'DONNELL M.J., DOW J.A.T., HUESMANN G.R., TUBLITZ N.J. and MADDRELL S.H.P. (1996). Separate control of anion and cation transport in Malpighian tubules of Drosophila melanogaster. J. Exp. Biol. 197: 421-428.

ORO A.E., MCKEOWN M. and EVANS RM (1990). Relationship between the product of Dosophila ultraspiracle locus and vertebrate retinoid $\mathrm{X}$ receptor. Nature 347: 298-301.

PATEL N.H (1994). Imaging neuronal subsets and other cell types in whole-mount Drosophila embryos and larvae using antibody probes. In Methods in Cell Biology, (Eds. L.S.B. Goldstein and E.A. Fyrberg) Academic Press Inc. 44: 445-
487.

PUTZ M., KESPER D.A., BUTTGEREIT D., RENKAWITZ-POHL R. (2005). In Drosophila melanogaster, the Rolling pebbles isoform 6 (Rols6) is essential for proper Malpighian tubule morphology. Mech. Dev. 122: 1206-1207.

RIDIFFORD L.M (1993). Hormones and Drosophila development. In The development of Drosophila. (Ed. M. Bates and A. Martinez-Aria). Cold Horbor Laboratory press, New York. 899-940.

RORTH P., SZABCO K., BAILEY A., LAVERTY T., REHM J., RUBIN G.M., WEIGHMANN K., MILAN M., BENES V., ANSORGE W. and COHEN S.M. (1998). Systematic gain of function genetics in Drosophila. Development 125: 1043-1057.

RYERSE J.S. (1978). Ecdysone switches off fluid secretion at pupation in insect MTs. Nature 271: 745-756.

SCHUBIGER M., WADE A., CARNEY G.E., TRUMAN J.W and BENDER M. (1998) Drosophila EcR-B ecdysone receptor isoforms are required for larval molting and for neuron remodeling during metamorphosis. Development 125: 20532062.

SCHUBIGER M., TOMITA S., SUNG C., ROBINOW S. and TRUMAN J.W. (2003). Isoform specific control of gene activity in vivo by the Drosophila ecdysone receptor. Mech. Dev. 120: 909-918.

SOZEN M.A., ARMSTRONG J.D., YANG M., KAISER K and DOW J.A.T. (1997). Functional domains are specified to single cell resolution in a Drosophila epithelium. Proc. Nat. Assoc. Sci. USA 94: 5207-5212.

SINGH S.R., LIU W and HOU S. (2007). The adult DrosophilaMalpighian tubule are maintained by pluripotent stem cells. Cell Stem Cell. 16: 191-203.

SUNG, C. and ROBINOW, S. (2000). Characterization of the regulatory elements controlling neuronal expression of the A-isoform of the ecdysone receptor gene of Drosophila melanogaster. Mech Dev 91: 237- 248.

TALBOT, W.S., SWYRYD, E.A. and HOGNESS, D.S. (1993). Drosophila tissues with different metamorphic responses to ecdysone express different ecdysone receptor isoforms. Cell. 73: 1323-1337

THOMAS, H.E., STUNNENBERG H.G. and STEWART A.F. (1993). Hetero dimerization of Drosophilaecdysone receptor with retinoid $X$ receptor and ultraspiracle. Nature 362: 471-475.

THUMMEL, C.S., BURTIS, K.C. and HOGNESS D.S. (1990). Spatial and tempora patterns of E74 transcription during Drosophila development. Ce//61:101-111

TRUMAN, J.W., TALBOT, W.S., FAHRBACH, S.E. and HOGNESS D.S. (1994) Ecdysone receptor expression in the CNS correlates with stage-specific responses to ecdysteroids during Drosophila and Manduca development. Deve/opment 120: 219-234.

WESSING, A and EICHELBERG, D. (1978). Malpighian tubules, rectal papillae and excretion. In Genetics and Biology of Drosophila (Eds. A. Ashburner and T.R.F Wright). London Academic Press, London, pp1-42.

YAO, T.P., SEGRAVES, W.A., ORO, A.E., McKEOWN, M. and EVANS, R.M. (1992). Drosophila ultraspiracle modulates ecdysone receptor function via heterodimer formation. Cel/71: 63-72. 


\section{Further Related Reading, published previously in the Int. J. Dev. Biol.}

See Special Issue Pattern Formation edited by Michael K. Richardson and Cheng-Ming Chuong at: http://www.ijdb.ehu.es/web/contents.php?vol=53\&issue=5-6

\section{Epithelial-Mesenchymal Transitions in development and disease: old views and new} perspectives

M. Angela Nieto

Int. J. Dev. Biol. (2005) 52: 2410-2410

Regulation of cell adhesion and migration in lens development

Peggy S. Zelenka

Int. J. Dev. Biol. (2004) 48: 857-865

Ventral nerve cord remodeling in a stingless bee (Melipona quadrifasciata anthidioides, Hymenoptera, Apidae) depends on ecdysteroid fluctuation and programmed cell death

Lucimara Z. Pinto, Marcela A. F. B. Laure, Marcia M. G. Bitondi, K. Hartfelder and Zila L. P. Simoes

Int. J. Dev. Biol. (2003) 47: 385-388

Regulation of cell migration during tracheal development in Drosophila melanogaster V. Petit, C. Ribeiro, A. Ebner and M. Affolter Int. J. Dev. Biol. (2002) 46: 125-132

Integrin regulatory switching in development: oscillation of beta 5 integrin mRNA expression during epithelial-mesenchymal interactions in tooth development

S. Yamada, K. M. Yamada and K. E. Brown Int. J. Dev. Biol. (1994) 38: 553-556

5 yr ISI Impact Factor $(2008)=3.271$

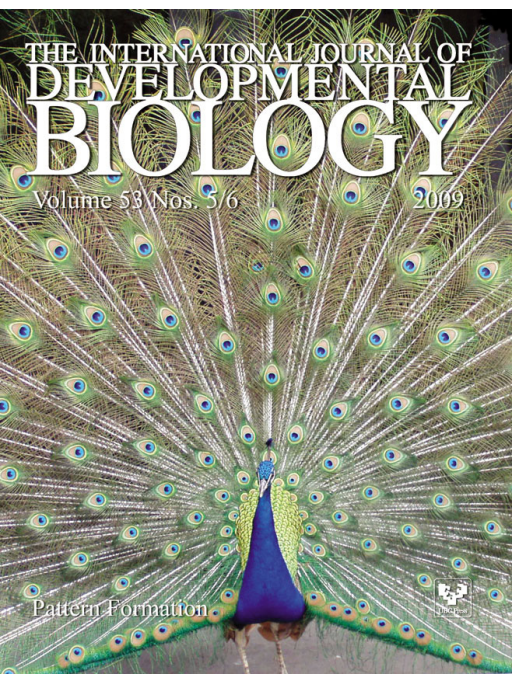

